\title{
Composição mineral de folhas de Oliveira (Olea europaea L.) em sistema de produção convencional
}

VIEIRA NETO, J.. ${ }^{*}$; SILVA, L.F.O. ${ }^{2}$; GONÇALVES, P.A.S. ${ }^{1}$; ZAMBON, C.R. ${ }^{3}$; VILLA, F. ${ }^{4}$

${ }^{1}$ Empresa de Pesquisa Agropecuária e Extensão Rural de Santa Catarina (EPAGRI), Estrada Geral, 453, Águas Negras, CEP: 88400-000, Ituporanga-Brasil "joaoneto@epagri.sc.gov.br ${ }^{2}$ Universidade Federal de Lavras (UFLA), Departamento de Agricultura, Caixa Postal: 3037, CEP: 37200-000, Lavras-Brasil ${ }^{3}$ Empresa de Pesquisa Agropecuária de Minas Gerais (EPAMIG), Rua Washington Alvarenga Viglione, s/ $n^{\circ}$, Bairro Vargedo, Caixa Postal: 28, CEP: 37517-000, Maria da Fé-Brasil ${ }^{4}$ Universidade Estadual do Oeste do Paraná (UNIOESTE), Centro de Ciências Agrárias, Rua Pernambuco, 1777, Centro, CEP: 85960-000, Marechal Candido Rondon-Brasil

RESUMO: Objetivou-se com este trabalho quantificar a composição mineral das folhas de Oliveira a fim de diagnosticar o estado nutricional das plantas cultivadas em sistema convencional. $O$ experimento foi conduzido com sete cultivares Arbequina, MGS Asc 315, MGS Mariense, MGS Grap 541, MGS Grap 561, Grappolo 575 e Leccino, em blocos casualizados com 4 repetições e parcelas com cinco plantas. Em maio de 2010 foram coletadas 60 folhas de cada cultivar (12 por planta) e submetidas a analise foliar. As cultivares apresentaram diferenças significativas entre si quanto aos teores foliares médios de nutrientes. Observou-se também que apesar da analise de solo apontar níveis elevados de Fe e Mn, a análise foliar acusou deficiência para esses elementos em todas as cultivares de Oliveira avaliadas. Os resultados alertam para os altos níveis de $\mathrm{Cu}$, como consequência do uso abusivo de fungicidas a base de oxicloreto e calda bordalesa nos pomares. A concentração elevada desse elemento nas folhas pode causar fitotoxidez às plantas. Desta forma, sugere-se o uso racional de fertilizantes e de produtos fitossanitários no cultivo da oliveira a fim de evitar fitotoxidez ou deficiências nutricionais.

Palavras-chave: nutrição mineral, toxidez, deficiência mineral.

ABSTRACT: Mineral composition of olive (Olea europaea L.) leaves in conventional production system. This study aimed to quantify the mineral composition of olive leaves to diagnose nutritional status of plants grown in conventional system. The experiment was conducted with seven cultivars: Arbequina, MGS Asc 315, MGS Mariense, MGS Grap 541, MGS Grap 561, Grappolo 575 and Leccino, in a randomized block design with four replications and five plants perplot. In May 2010, we collected 60 leaves for each cultivar (12 per plant), whichwere dried and subsequently analyzed. The results advert to the high levels of $\mathrm{Cu}$, a consequence of the excessive use of fungicides based on copper oxychloride and Bordeaux mixture in the orchards. The high concentration of this element in the leaves can bephytotoxicity to plants and harmful to human health, since the leaves are used popularly as an aid in procedures for weight loss. Thus, we suggest the rational use of fertilizers and pesticides in the cultivation of olive trees to prevent nutritional deficiency or phytotoxicity and, if the research acknowledges the phytotherapic effect of leaves, the adoption of crops in the differentiated system of production, to obtain raw material with good quality and suitable for consumption in natura.

Keywords: mineral nutrition, toxicity, mineral deficiency

\section{INTRODUÇÃO}

Estudos sobre fertilização em olivais e de metodologia de análise foliar são importantes para determinar os níveis críticos de nutrientes em folhas de oliveira (Beutel et. al, 1983).

A diagnose foliar permite conhecer o estado nutricional dos tecidos, o qual está relacionado diretamente com a taxa de crescimento ou de produção (Jones et al., 1990). Dessa correlação são estabelecidos valores para os teores de nutrientes que correspondem a mudanças fisiológicas 
essenciais. Esses pontos representam níveis críticos e delimitam faixas de concentrações de deficiências nutricionais, de níveis adequados ou de toxidez de minerais (Marinho et al., 2002).

Atualmente a análise foliar é considerada uma importante ferramenta para a avaliação do estado nutricional de frutíferas em geral, pois interage com todos os fatores que influenciam o estado nutritivo da planta (Fernández-Escobar, 1999). Em consequência, se aplicará um elemento nutritivo somente se a análise foliar indicar seu valor abaixo do adequado, ou seja, quando existe a deficiência deste elemento.

Em Minas Gerais e no Brasil existem carências de tecnologias aplicadas a expansão da olivicultura, sendo importante estudar, entre outros aspectos, o manejo de sua fertilidade e nutrição.

Segundo Larcher (2000), os principais nutrientes necessários ao desenvolvimento de uma planta, devem estar prontamente disponíveis desde os estágios iniciais de crescimento, a fim de promover o desenvolvimento máximo, evitando assim que ocorram desequilíbrios entre o conteúdo mineral e o incremento da matéria seca.

Em virtude dos poucos estudos com essa espécie, principalmente no que se refere ao manejo da fertilidade e nutrição da planta, a expansão do cultivo da olivicultura nacional tem ocorrido em ritmo lento. Nesse sentido, objetivou-se neste trabalho quantificar a composição mineral de folhas de oliveira, a fim de diagnosticar o estado nutricional das plantas cultivadas em sistema de produção convencional, em Minas Gerais.

\section{MATERIAL E MÉTODO}

$O$ estudo foi conduzido em um pomar de oliveira com dois anos de idade, pertencente à Empresa de Pesquisa Agropecuária de Minas Gerais (EPAMIG), no município de Maria da Fé, na

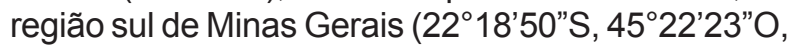
altitude $1276 \mathrm{~m}$ ).
O pomar foi constituído por sete cultivares de oliveira: Arbequina; MGS Asc 315; MGS Mariense; MGS Grap 541; MGS Grap 561; Grappolo 575 e Leccino, em blocos casualizados com 4 repetições e parcelas com cinco plantas, espaçadas em $6 \mathrm{x}$ $4 \mathrm{~m}$. A área experimental recebeu os tratamentos culturais e fitossanitários preconizados para cultivo convencional da cultura, de acordo com Mesquita et al. (2006), Pereira et. al. (2006) e Prado \& Silva (2006).

As características químicas do solo, classificado como Argissolo Vermelho (EMBRAPA, 2006), por ocasião do diagnóstico foliar, são apresentadas na Tabela 1.

$\mathrm{Na}$ segunda quinzena de maio de 2010 , período em que a concentração de nutrientes nas folhas é mais estável, foram coletadas amostras de folhas das oliveiras. Para cada cultivar foram coletadas 60 folhas com pecíolo por parcela (12 folhas por planta), na parte central dos ramos do ano localizados na porção mediana da copa (Mesquita et al., 2006).

As folhas foram lavadas com água deionizada e postas para secar em estufa de circulação de ar forçada, a $65^{\circ} \mathrm{C}$, até atingir peso constante, em seguida foram moídas em moinho tipo Wiley, passadas em peneira de 20 mesh e homogeneizadas. A determinação do $P$ foi realizada por colorimetria com vanadato-molibdato de amônio (cor amarela) em espectrofotômetro UVI VIS (Silva, 1999). O K e o Na foram determinados por fotometria de emissão (Perkin-Elmer, 1976) e os elementos, $\mathrm{Ca}, \mathrm{Mg}, \mathrm{S}, \mathrm{Fe}, \mathrm{Mn}, \mathrm{Cu}, \mathrm{Zn}$ e Al por espectrofotometria de absorção atômica (PerkinElmer, 1976). O B foi determinado pelo método de colorimetria usando azometina - H (Silva, 1999).

O nível de concentração foliar de cada nutriente avaliado foi classificado em deficiente, adequado e tóxico, para cada cultivar, pelo critério descrito por Mesquita et al. (2006) e Esprazzato (2008) (Tabela 2).

O teste $\mathrm{t}$ de Student, ao nível de $5 \%$ de

TABELA 1. Análise do solo por ocasião do diagnóstico foliar. EPAMIG, Maria da Fé, 2010.

\begin{tabular}{|c|c|c|c|c|c|c|c|c|c|c|}
\hline $\mathrm{pH}\left(\mathrm{H}_{2} \mathrm{O}\right)$ & $\mathrm{P}$ & K & $\mathrm{Na}$ & $\mathrm{Ca}$ & $\mathrm{Mg}$ & $\mathrm{Al}$ & $\mathrm{H}+\mathrm{Al}$ & SB & $\mathrm{t}$ & $\mathrm{T}$ \\
\hline \multirow{2}{*}{6,1} & \multicolumn{2}{|c|}{$-\mathrm{mg} \mathrm{dm}^{-3}-$} & \multicolumn{8}{|c|}{ - $\mathrm{cmol}_{\mathrm{c}} \mathrm{dm}^{-3}$} \\
\hline & 120 & 178 & - & 4,8 & 0,8 & 0,0 & 2,1 & 6,1 & 6,1 & 8,2 \\
\hline MO & \multicolumn{2}{|l|}{$\mathrm{B}$} & $\mathrm{Cu}$ & \multicolumn{2}{|c|}{$\mathrm{Fe}$} & $\mathrm{Mn}$ & $\mathrm{Zn}$ & $S$ & $\mathrm{~m}$ & V \\
\hline \multicolumn{3}{|l|}{ dag $\mathrm{kg}^{-1}$} & \multicolumn{6}{|c|}{ ב-1 } & \multicolumn{2}{|c|}{---------\%-------- } \\
\hline 2,5 & 0,3 & & 12,0 & & & 14,3 & 1,9 & 35,3 & 0 & 74,3 \\
\hline
\end{tabular}

$\mathrm{SB}$ = Soma de bases trocáveis; $\mathrm{t}=$ Capacidade de troca catiônica efetiva; $\mathrm{T}$ = Capacidade de troca catiônica a pH 7,0; $\mathrm{m}=$ Índice de saturação de alumínio, V= Índice de saturação de bases. 
TABELA 2. Níveis de macro e micronutrientes adotados na análise foliar de plantas de oliveira. EPAMIG, Maria da Fé, 2010.

\begin{tabular}{cccc}
\hline Elemento & Deficiente & Adequado & Tóxico \\
\hline $\mathrm{N}($ dag kg-1) & 1,4 & $1,5-2,0$ & - \\
$\mathrm{P}($ dag kg-1) & 0,05 & $0,1-0,3$ & - \\
$\mathrm{K}($ dag kg-1) & 0,4 & 1,0 & - \\
$\mathrm{Ca}($ dag kg-1) & 0,3 & 2,0 & - \\
$\mathrm{Mg}\left(\right.$ dag kg $\left.^{-1}\right)$ & 0,08 & 0,6 & - \\
$\mathrm{S}\left(\right.$ dag kg $\left.^{-1}\right)$ & $<0,1$ & $0,15-0,2$ & $>0,25$ \\
$\mathrm{Na}\left(\right.$ dag kg $\left.^{-1}\right)$ & - & - & $>0,2$ \\
$\mathrm{~B}\left(\mathrm{mg} \mathrm{kg}^{-1}\right)$ & 14 & $19-150$ & 185 \\
$\mathrm{Cu}\left(\mathrm{mg} \mathrm{kg}^{-1}\right)$ & - & $4-5$ & - \\
$\mathrm{Mn}\left(\mathrm{mg} \mathrm{kg}^{-1}\right)$ & - & 20 & - \\
$\mathrm{Zn}\left(\mathrm{mg} \mathrm{kg}^{-1}\right)$ & - & $10-32$ & - \\
$\mathrm{Fe}\left(\mathrm{mg} \mathrm{kg}^{-1}\right)$ & $<50$ & $90-180$ & $>220$ \\
\hline
\end{tabular}

Fonte: Adaptado de Mesquita et al. (2006) e Esprazzato (2008).

probabilidade de erro, foi utilizado para comparar as médias amostrais das concentrações de nutrientes com seus respectivos níveis críticos. A análise foi realizada através do programa estatístico NTIA (Embrapa, 1997).

\section{RESULTADO E DISCUSSÃO}

As concentrações de $\mathrm{N}, \mathrm{P}, \mathrm{k}, \mathrm{Ca}$ e $\mathrm{S}$ (Tabela 3), B, Zn (Tabela 4) nas folhas de oliveira ocorreram dentro dos níveis adequados em todas as cultivares, conforme indicação da Tabela 2. Em estudo realizado em Maria da Fé, Sul de Minas, Mesquita et al. (2012) também encontram níveis foliares desses elementos, em plantas de oliveira, dentro da normalidade.

$\mathrm{O} \mathrm{Mg}$ foi deficiente nas folhas das cultivares Arbequina e MGS Mariense (Tabela $3)$, mesmo com teor médio no solo $\left(0,8 \mathrm{cmol}_{\mathrm{c}} \mathrm{dm}^{-}\right.$ $\left.{ }^{3}\right)$. A deficiência desse elemento, mesmo sendo móvel na planta, advém principalmente da alta concentração de K no solo (Malavolta, 2006).

Embora o resultado da análise do solo (Tabela 1) da área experimental, na ocasião da amostragem foliar, tenha acusado níveis elevados Fe $\left(58,2 \mathrm{mg} \mathrm{dm}^{-3}\right)$ e $\mathrm{Mn}\left(14,3 \mathrm{mg} \mathrm{dm}^{-3}\right)$, o nível foliar desses elementos foi deficiente em todas as cultivares (Tabela 4).

A deficiência de $\mathrm{Fe}$ e $\mathrm{Mn}$ no tecido foliar, mesmo estando abundantes no solo, está associada à sua baixa mobilidade na planta e ao desequilíbrio que apresentam entre si, pelo efeito de inibição competitiva. Para o Fe, em especial, o excesso de $P$ no solo é mais um agravante.

Através da diagnose foliar também foi possível detectar níveis de toxidez para $\mathrm{Na}$ e $\mathrm{Cu}$ (Tabelas 3 e 4). A concentração de Na nas folhas de oliveira superou em 3 vezes o nível de toxidez $\left(0,2 \mathrm{dag} \mathrm{kg}^{-1}\right)$. Para o $\mathrm{Cu}$, esta discrepância foi ainda maior alcançando teores 33 vezes maiores do que o nível adequado $\left(4 \mathrm{mg} \mathrm{kg}^{-1}\right)$. Mesquita et al. (2012) encontraram níveis foliares de Cu em plantas de oliveira variando entre 11 a 38 vezes acima do nível adequado. O sódio não foi avaliado pelos autores no do Sul de Minas.

TABELA 3. Média e classificação da concentração foliar de macronutriente mais sódio em deficiente (D), adequado (A) e tóxico (T) em oliveira. EPAMIG, Maria da Fé, 2010.

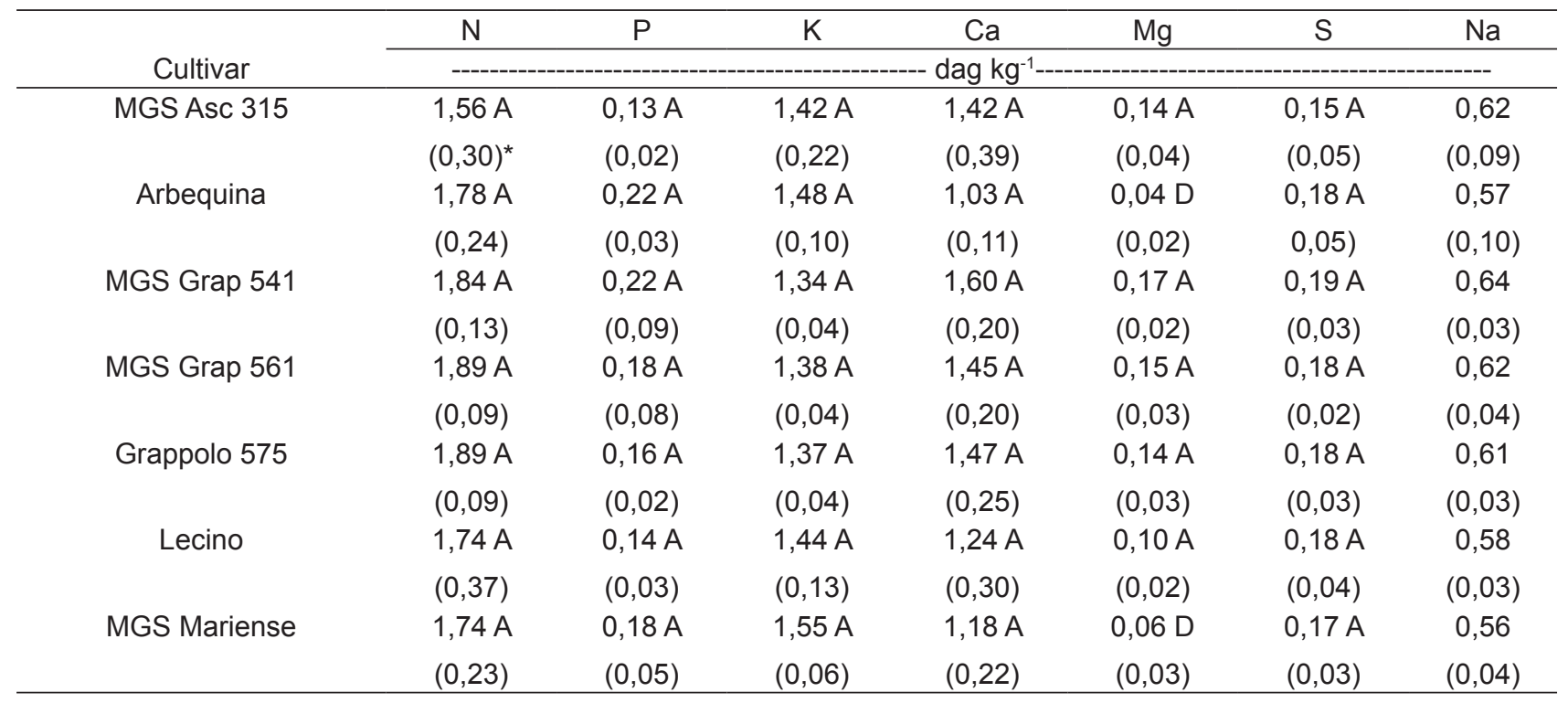

* Semiamplitude do intervalo de confiança para a média com base na distribuição de te Student com $95 \%$ de confiança. 
TABELA 4. Média e classificação da concentração foliar de micronutriente em deficiente (D), adequado (A) e tóxico (T) em oliveira. EPAMIG, Maria da Fé, 2010.

\begin{tabular}{|c|c|c|c|c|c|}
\hline \multirow[b]{2}{*}{ Cultivar } & $\mathrm{B}$ & $\mathrm{Cu}$ & $\mathrm{Mn}$ & $\mathrm{Zn}$ & $\mathrm{Fe}$ \\
\hline & \multicolumn{5}{|c|}{ ר } \\
\hline \multirow[t]{2}{*}{ MGS Asc 315} & $31,70 \mathrm{~A}$ & $127,83 \mathrm{~T}$ & $12,23 \mathrm{D}$ & $22,60 \mathrm{~A}$ & $57,13 \mathrm{D}$ \\
\hline & $(8,15)^{\star}$ & $(37,64)$ & $(4,27)$ & $(6,89)$ & $(23,78)$ \\
\hline \multirow[t]{2}{*}{ Arbequina } & $25,30 \mathrm{~A}$ & $99,83 \mathrm{~T}$ & $9,25 \mathrm{D}$ & $25,82 \mathrm{~A}$ & $55,35 \mathrm{D}$ \\
\hline & $(4,36)$ & $(29,13)$ & $(2,61)$ & $(5,31)$ & $(6,79)$ \\
\hline \multirow[t]{2}{*}{ MGS Grap 541} & $36,75 \mathrm{~A}$ & $120,05 \mathrm{~T}$ & $13,05 \mathrm{D}$ & $19,53 \mathrm{~A}$ & $60,33 \mathrm{D}$ \\
\hline & $(8,23)$ & $(20,49)$ & $(3,44)$ & $(5,68)$ & $(24,41)$ \\
\hline \multirow[t]{2}{*}{ MGS Grap 561} & $35,50 \mathrm{~A}$ & $124,15 \mathrm{~T}$ & $13,73 \mathrm{D}$ & $34,02 \mathrm{~A}$ & $60,73 \mathrm{D}$ \\
\hline & $(7,00)$ & $(47,59)$ & $(0,27)$ & $(5,63)$ & $(23,48)$ \\
\hline \multirow[t]{2}{*}{ Grappolo 575} & $36,55 \mathrm{~A}$ & $133,95 \mathrm{~T}$ & $15,08 \mathrm{D}$ & $36,20 \mathrm{~A}$ & $54,20 \mathrm{D}$ \\
\hline & $(5,88)$ & $(38,99)$ & $(4,36)$ & $(11,10)$ & $(10,34)$ \\
\hline \multirow[t]{2}{*}{ Lecino } & $28,82 \mathrm{~A}$ & $104,70 \mathrm{~T}$ & $11,43 \mathrm{D}$ & $21,70 \mathrm{~A}$ & $53,40 \mathrm{D}$ \\
\hline & $(6,41)$ & $(36,56)$ & $(3,83)$ & $(2,98)$ & $(36,62)$ \\
\hline \multirow[t]{2}{*}{ MGS Mariense } & $30,30 \mathrm{~A}$ & $90,98 \mathrm{~T}$ & $13,15 \mathrm{D}$ & $22,80 \mathrm{~A}$ & $53,88 \mathrm{D}$ \\
\hline & $(9,40)$ & $(15,67)$ & $(4,26)$ & $(5,08)$ & $(17,75)$ \\
\hline
\end{tabular}

* Semiamplitude do intervalo de confiança para a média com base na distribuição de t de Student com $95 \%$ de confiança.

$\mathrm{O}$ uso do Cu como fungicida é frequente na agricultura, em especial na fruticultura, o que pode explicar os significativos níveis observados. Fungicidas a base de oxicloreto de cobre têm sido usados com frequência pelos olivicultores, embora não estejam registrados no Ministério da Agricultura, Pecuária e Abastecimento (MAPA) para a cultura. Além destes, é comum o uso de preparados químicos antifúngicos conhecidos como calda bordalesa e calda viçosa para a proteção de plantas, ambos com sulfato de cobre em sua composição (Oliveira et al., 2009).

Esses produtos, quando usados indiscriminadamente, podem ocasionar ação fitotóxica às plantas, manifestada por meio de redução do crescimento, queda de flores e frutos, crestamento, redução da fotossíntese e menor produção (Peruch \& Bruna, 2008). O meio ambiente e consequentemente a saúde do homem também são afetados, pois alguns fungicidas não são biodegradáveis, ou se decompõem a longo prazo (Faria et al., 2007).

A elevada concentração de Cu nas folhas da oliveira é particularmente preocupante, pois as folhas de oliveira vêm sendo popularmente utilizadas como auxiliar em processos de emagrecimento, principalmente para a redução da gordura abdominal (Contreras, 2011; Oliveira, 2011; Chá \& Cia, 2011). As folhas de oliveira são usadas em vários países na dieta humana, como extrato, chá, pó, pelo potencial de compostos bioativos, que podem ter efeitos antioxidante, anti-hipertensivos, aterogênicos, propriedades anti-inflamatórias, hipoglicêmicos e hipocolesterolêmicos (EI \& Karakaya, 2009). No entanto, estudos científicos sobre suas propriedades terapêuticas são escassos, bem como não há no Sistema Brasileiro de Legislação de Saúde norma regulatória do uso de folhas de oliveira para esse fim (Ministério da Saúde, 2011a). De fato, a espécie Olea europaea nem mesmo consta na RENISUS - Relação Nacional de Plantas Medicinais de Interesse ao Sistema Único de Saúde (SUS), cuja finalidade é orientar pesquisas e estudos sobre o tema para garantir à população brasileira o acesso seguro e o uso racional de plantas medicinais e fitoterápicos (Ministério da Saúde, 2011b).

O sódio, mesmo estando acima do nível de toxidez $\left(0,2\right.$ dag $\left.\mathrm{kg}^{-1}\right)$, não chega a representar um risco à saúde, uma vez que a concentração média encontrada nas folhas $\left(0,60 \mathrm{mg} \mathrm{kg}^{-1}\right)$ está bem abaixo de valores encontrados na maioria dos alimentos industrializados (Rique et al., 2002).

Com base nos resultados observados nesse trabalho sugere-se o uso racional de fertilizantes e de produtos fitossanitários no cultivo convencional da oliveira a fim de evitar fitotoxidez ou deficiências nutricionais.

Além disso, caso as pesquisas reconheçam o efeito fitoterápico das folhas de oliveira, será necessário um sistema de cultivo diferenciado visando à obtenção de matéria prima com qualidade e própria para o consumo in natura. 


\section{REFERÊNCIA}

BEUTEL, J., URIU, K.; LILLELAND, O. 1983. Leaf analysis for California deciduous fruits. In: REISENAUER, H.M. (Ed.). Soil and plant tissue testing in California. Publishers, University of California Bul, 1897. p. 15-17.

CHÁ \& CIA. Ervas Medicinais, Disponível em: <http:// www.chaecia.com.br/loja/produto-111058-1297oliveira_olea_europaea_I_100_grg?gclid=CNfOn3tKcCFRJY2godc0jLBg> Acesso em: 04 mar. 2011.

CONTRERAS, E. As novas pílulas naturais para emagrecer, Disponível em: http://boaforma.abril. com.br/dieta/aliados-da-dieta/novas-pilulas-naturaisemagrecer-510459.shtml>. Acesso em: 22 mar. 2011.

EL, S.N.; KARAKAYA, S. Olive tree (Olea europaea) leaves: potential beneficial effects on human health. Nutrition Reviews, v.67, n.11, p.632-638, 2009.

EMBRAPA. Centro Nacional e Pesquisa em Solos. Sistema brasileiro de classificação de solos. Brasilia: Embrapa-SPI; Rio de Janeiro: Embrapa-Solos, 2006. $306 \mathrm{p}$.

Centro Nacional de Pesquisa Tecnológica em Informática para a Agricultura. Ambiente de software NTIA, versão 4.2.2: manual do usuário - ferramental estatístico. Campinas: EMBRAPA/CNPTIA, 1997. 258p.

ESPRAZZATO, C.A. Apuntes de olivicultura. Mendoza: Agrositio libros, 2008. 152p.

FARIA, N.M.X.; FASSA, A.G.; FACCHINI, L.A. Intoxicação por agrotóxicos no Brasil: os sistemas oficiais de informação e desafios para realização de estudos epidemiológicos. Ciência \& Saúde Coletiva, n.12, v.1, p.25-38, 2007.

FERNÁNDEZ-ESCOBAR, R., MORENO, R., GARCÍACREUS, M. Seasonal changes of mineral nutrients in olive leaves during the alternate-bearing cycle. Science Horticulturae, v.82, p.25-45, 1999.

JONES, J.B.; ECK, H.V.; VOSS, R. Plant analysis as an aid in fertilizing corn and grain sorghum. In: WESTERMAN, R. L. (Ed.). Soil testing and plant analysis. Madison: SSSA, 1990. p. 521-549.

LARCHER, W. Ecofisiologia vegetal. São Carlos: Rima Artes e Textos, 2000.531p.

MALAVOLTA, E. Manual de nutrição mineral de plantas. São Paulo: Ceres, 2006. 638p.

MARINHO, C.S.; MONNERAT, P.H.; CARVALHO, A.J.R.C.; MARTINS, S.L.D.; VIEIRA, A. Análise química do pecíolo e limbo foliar como indicadora do estado nutricional dos mamoeiros solo e formosa. Scientia Agricola, Piracicaba, v.59, n.2, p.373-381, 2002.

MESQUITA, H.A.de; FRÁGUAS, J.C. PAULA, M.B. Adubação e nutrição da oliveira. Informe Agropecuário. Azeitonas e azeite de oliva tecnologias de produção Belo Horizonte v.27, n.231, p. 68-72, 2006.

MESQUITA, H.A.de; GARCIA, C.N.; COSTA, E.L.da. Solos, aspectos nutricionais e sugestões de fertilização. In: OLIVEIRA, A.F.de. (Org.). Oliveira no Brasil: tecnologias de produção. Belo Horizonte: EPAMIG, 2012. p. 311-348.

MINISTÉRIO DA SAÚDE. Sistema de Legislação da Saúde, Disponível em: <http://portal2.saude.gov.br/ saudelegis/leg_norma_pesq_consulta.cfm>. Acesso em: 04 mar. 2011a.

Fitoterapia, Disponível em: <http://portal.saude. gov.br/portal/arquivos/pdf/RENISUS_2010.pdf>. Acesso em: 04 mar. 2011b.

OLIVEIRA, A. Continuando sobre os fitoterápicos, Disponível em: <http://boaforma.abril.com.br/blog/ corpo-novo-vida-nova-andrea-oliveira/2010/05/07/ continuando-sobre-os-fitoterapicos/>. Acesso em: 22 mar. 2011.

.; VIEIRA NETO, J.; GONÇALVES, E.D.; MESQUITA, H.A.de Pioneirismo marca pesquisa sobre oliveira em Minas Gerais. Informe Agropecuário, v.30, p.109-117, 2009.

PEREIRA, L.V.; OLIVEIRA, A.F.de; CARVALHO,V.L.; SOUZA, P.E. Principais doenças da oliveira: descrição e danos. Informe Agropecuário, v.27, n.231, p.73-78, 2006.

PERKIN-ELMER. Analytical methods for atomic absorption spectrophotometry. Norwalk: Perkin Elmer Corporation, 1976. 476p.

PERUCHI, L.A.M.; BRUNA, E.D. Relação entre doses de calda bordalesa e de fosfito potássico na intensidade do míldio e na produtividade da videira cv. 'Goethe'. Ciência Rural, Santa Maria, v.38, n.9, p.2413-2418, 2008.

PRADO, E.; SILVA, R.A. Principais pragas da oliveira: biologia e manejo. Informe Agropecuário, v.27, n.231, p.79-83, 2006.

RIQUE, A.B.R.; SOARES, E.A.; MEIRELLES, C.M. Nutrição e exercício na prevenção e controle das doenças cardiovasculares. Revista Brasileira de Medicina do Esporte, v.8, n.6, p.244-254, 2002.

SILVA, F.C. Manual de análises químicas de solos, plantas e fertilizantes. Brasília: Embrapa Solos, 1999. $370 \mathrm{p}$.

Rev. Bras. PI. Med., Campinas, v.16, n.1, p.122-126, 2014. 